\title{
Sel $\mathrm{f}$ - or gani zat i on mechani sm i $\mathrm{n}$ t wo- di mensi onal poi nt vortex system
}

\begin{tabular}{|l|l|}
\hline 著者 & Yat suyanagi Yui chi , Hat or i Tadat sugu \\
\hline $\begin{array}{l}\text { j our nal or } \\
\text { publ i cat } \mathrm{i} \text { on t i t l e }\end{array}$ & Al P Conf er ence Proceedi ngs 1668 \\
\hline vol une & 1668 \\
\hline page range & 050003 \\
\hline year & 2015 \\
\hline 出版者 & Aner i can I nst i tute of Physi cs \\
\hline 権利 & ( C) 2015 Al P Publ i shi ng LLC \\
\hline URL & ht t p: //hdl . handl e. net /10297/9167 \\
\hline
\end{tabular}




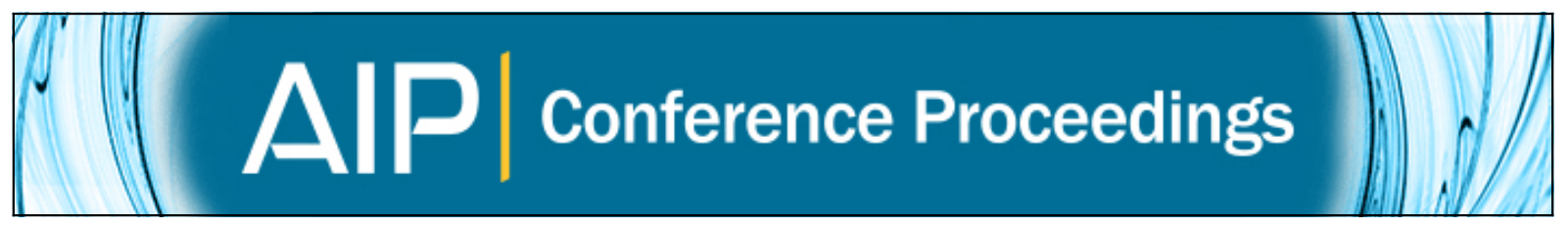

\section{Self-organization mechanism in two-dimensional point vortex system}

Yuichi Yatsuyanagi and Tadatsugu Hatori

Citation: AIP Conference Proceedings 1668, 050003 (2015); doi: 10.1063/1.4923122

View online: http://dx.doi.org/10.1063/1.4923122

View Table of Contents: http://scitation.aip.org/content/aip/proceeding/aipcp/1668?ver=pdfcov

Published by the AIP Publishing

Articles you may be interested in

Self-organized two-dimensional onions

Appl. Phys. Lett. 94, 113507 (2009); 10.1063/1.3101373

Relaxation and self-organization in two-dimensional plasma and neutral fluid flow systems

Phys. Plasmas 15, 022308 (2008); 10.1063/1.2844441

Characteristics of two-dimensional turbulence that self-organizes into vortex crystals

AIP Conf. Proc. 498, 85 (1999); 10.1063/1.1302105

Properties of two-dimensional electron gas containing self-organized quantum antidots

Appl. Phys. Lett. 75, 2942 (1999); 10.1063/1.125195

On the symmetry of self-organized structures in two-dimensional turbulence

Phys. Fluids 7, 2108 (1995); 10.1063/1.868461 


\title{
Self-Organization Mechanism in Two-Dimensional Point Vortex System
}

\author{
Yuichi Yatsuyanagi* and Tadatsugu Hatori ${ }^{\dagger}$ \\ *Faculty of Education, Shizuoka University, Shizuoka 422-8529, Japan \\ ${ }^{\dagger}$ National Institute for Fusion Science
}

\begin{abstract}
A kinetic equation for a two-dimensional double-species point vortex system is obtained. The obtained collision term consisting of a diffusion term and a drift term has several physically good properties, for example, conserving a mean field energy and satisfying $\mathrm{H}$ theorem. It should be emphasized that the drift term plays an essential role for self-organization in a point vortex system with negative temperature.
\end{abstract}

Keywords: Self-organization, point vortex system, kinetic equation, negative tempearture

PACS: $05.65 .+b, 47.32 . c-, 05.20 . \mathrm{Dd}$

\section{INTRODUCTION}

In this paper, we will present a kinetic equation for the two-dimensional (2D) point vortex system. The collision term of the kinetic equation is composed of a diffusion term and a drift term. It is revealed that the drift term plays an essential role in self-organization in a $2 \mathrm{D}$ point vortex system.

There are wide variety of physical and chemical systems where well organized spatial or temporal structures and functions arise spontaneously. This is called "self-organization" [1]. In pure electron plasma experiments, vortex crystal distributions are observed [2,3,4]. The electrons aggregate in small regions against their repulsive electrostatic force. Large-scale vortical structure formations are found in Jupiter's great red spot, typhoons, and so on. These phenomena are associated with the self-organization, which also corresponds to an "energy inverse cascade," which is well-known in 2D turbulence. In an energy inverse cascade, the energy is transferred from a small scale to a large scale. As a result, the large-scale vortex structure is maintained for a long time.

In this paper, we focus our attention to the self-organization of isolated vortices. Onsager introduced a concept of "negative temperature" to sketch a possible explanation for the self-organization of the vortex structure [5]. Joyce and Montgomery showed that an equilibrium distribution of the vortices at negative temperature is characterized by the sinh-Poisson equation [6]. The self-organization (clump formation) with like-sign vortices at negative temperature is numerically shown by, for example, Yatsuyanagi et. al. [7]. Montgomery et. al. showed that 2D Navier-Stokes fluid at a high Reynolds number relaxes to the sinh-Poisson state at negative temperature, although the sinh-Poisson equation only describes an equilibrium distribution of a discrete point vortex system at negative temperature. Since then, much research effort has been devoted to understanding the relaxation process in the negative temperature state leading to large-scale structure formation. For this purpose, we have proposed a kinetic theory for a single-species point vortex system with the Klimontovich formalism [8].

In this paper, we present a kinetic theory for a point vortex system with positive and negative vortices. The obtained kinetic equation evidences the important role of the drift term in the self-organization of a $2 \mathrm{D}$ point vortex system. The system with double-sign vortices elucidates the self-organization mechanism more clearly than the single-sign case.

\section{PLASMA KINETIC THEORY}

Kinetic equations describe a time evolution of a macroscopic system in terms of a probability density function. Hierarchy of the equations is shown in Fig. 1. The left-hand side class corresponds to the time-reversible microscopic equations, such as the Klimontovich equation and Liouville equation. The right-hand side class corresponds to the time-irreversible, macroscopic equations, such as the Navier-Stokes equation. The middle class corresponds to the intermediate-scale equations, in which the Boltzmann equation, Fokker-Planck equation and Vlasov equation are classified. They are referred to as kinetic equations. 
The Klimontovich equation is a microscopic equation having a discretized particle solution.

$$
\begin{array}{r}
\frac{\partial \hat{f}}{\partial t}+\vec{v} \cdot \vec{\nabla} \hat{f}+\frac{q}{m}(\hat{\vec{E}}+\vec{v} \times \hat{\vec{B}}) \cdot \frac{\partial \hat{f}}{\partial \vec{v}}=0, \\
\hat{f}(\vec{r}, \vec{v}, t)=\sum_{i} \delta\left(\vec{r}-\vec{r}_{i}(t)\right) \delta\left(\vec{v}-\vec{v}_{i}(t)\right)
\end{array}
$$

By replacing the microscopic variable $\hat{f}$ with a macroscopic variable which is defined by an ensemble average of the microscopic variable and a fluctuation, and averaging whole the equation, the following macroscopic equation with collisional effect is obtained.

$$
\frac{\partial f}{\partial t}+\vec{v} \cdot \vec{\nabla} f+\frac{q}{m}(\vec{E}+\vec{v} \times \vec{B}) \cdot \frac{\partial f}{\partial \vec{v}}=-\frac{q}{m}\left\langle(\delta \vec{E}+\vec{v} \times \delta \vec{B}) \cdot \frac{\partial \delta f}{\partial \vec{v}}\right\rangle
$$

This equation describes a time evolution of a system in terms of the continuous probability density function instead of the particle distribution. After some further manipulation, the Fokker-Planck equation is obtained.

$$
\begin{array}{r}
\frac{\partial f}{\partial t}+\vec{v} \cdot \vec{\nabla} f+\frac{q}{m}(\vec{E}+\vec{v} \times \vec{B}) \cdot \frac{\partial f}{\partial \vec{v}}=C(f) \\
C(f)=\frac{\partial}{\partial \vec{v}} \cdot\left(\mathrm{D} \cdot \frac{\partial f}{\partial \vec{v}}+\vec{A} f\right)
\end{array}
$$

When the system reaches an equilibrium state, balancing the diffusion term with the friction term yields zero collisional effect. In plasmas, long-range Coulomb interaction rather than the short-range collisions governs the whole dynamics of the system. For these systems the Vlasov equation is appropriate.

$$
\frac{\partial f}{\partial t}+\vec{v} \cdot \vec{\nabla} f+\frac{q}{m}(\vec{E}+\vec{v} \times \vec{B}) \cdot \frac{\partial f}{\partial \vec{v}}=0 .
$$

Note that the collisional effect in Vlasov Eq. is dropped by approximation.

\section{POINT VORTEX SYSTEM}

Similar to the Klimontovich equation, the two-dimensional inviscid Euler equation has a particle solution called the point vortex. The vorticity $\hat{\omega}(\vec{r}, t)$ is defined by a sum of the Dirac delta functions.

$$
\begin{aligned}
\hat{\omega}(\vec{r}, t) & =\hat{\omega}_{+}(\vec{r}, t)+\hat{\omega}_{-}(\vec{r}, t), \\
\hat{\omega}_{+} & =\sum_{i=1}^{N_{+}} \Omega_{0} \delta\left(\vec{r}-\vec{r}_{i}(t)\right), \\
\hat{\omega}_{-} & =-\sum_{i=N_{+}+1}^{N_{+}+N_{-}} \Omega_{0} \delta\left(\vec{r}-\vec{r}_{i}(t)\right)
\end{aligned}
$$

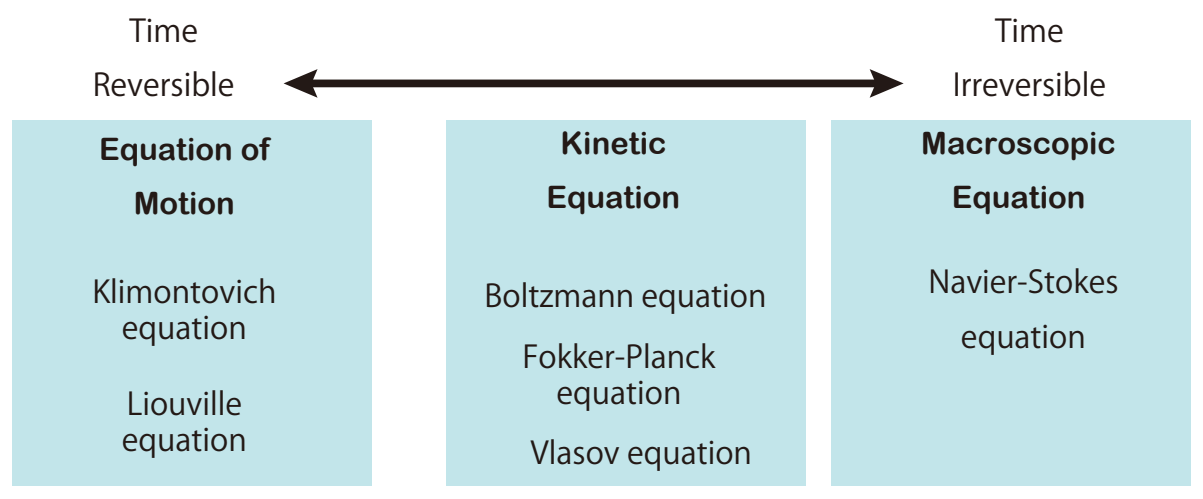

FIGURE 1. Hierarchy of the equations is shown. 


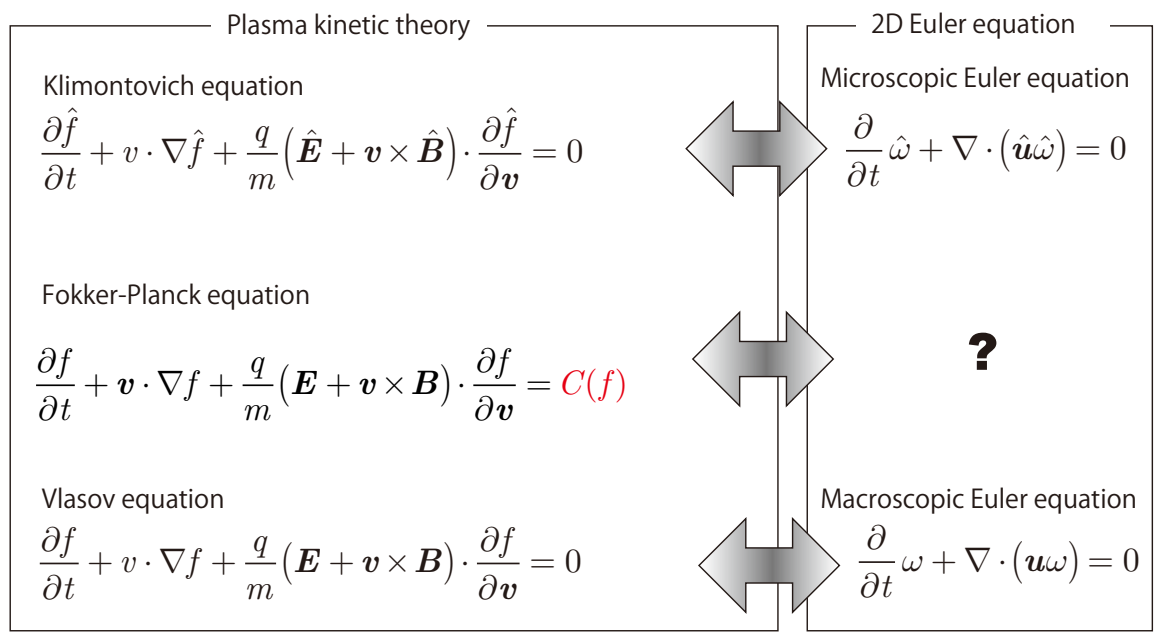

FIGURE 2. Correspondence of the plasma kinetic theory with $2 \mathrm{D}$ Euler equation is shown. There is no counterpart of the Fokker-Planck equation.

where $\Omega_{0}$ is a positive constant which determines circulation (strength) of each point vortex. The point vortex is a formal solution of the 2D Euler equation:

$$
\begin{aligned}
\frac{\partial}{\partial t} \hat{\omega}(\vec{r}, t) & =\frac{\partial}{\partial t}\left(\sum_{i} \Omega_{0} \delta\left(\vec{r}-\vec{r}_{i}(t)\right)\right) \\
& =-\sum_{i} \Omega_{0}\left(\frac{\partial}{\partial t} \vec{r}_{i}(t)\right) \cdot \vec{\nabla} \delta\left(\vec{r}-\vec{r}_{i}(t)\right) \\
& =-\sum_{i} \Omega_{0} \hat{\vec{u}}\left(\vec{r}_{i}(t), t\right) \cdot \vec{\nabla} \delta\left(\vec{r}-\vec{r}_{i}(t)\right) \\
& =-\hat{\vec{u}}(\vec{r}, t) \cdot \vec{\nabla} \sum_{i} \Omega_{0} \delta\left(\vec{r}-\vec{r}_{i}(t)\right) \\
& =-\hat{\vec{u}}(\vec{r}, t) \cdot \vec{\nabla} \hat{\omega}(\vec{r}, t)
\end{aligned}
$$

We assume that the 2D Euler equation having a point vortex solution is a microscopic equation as a solution of a macroscopic equation should be given by a smooth continuous function, instead of a discrete one.

$$
\frac{\partial \hat{\omega}}{\partial t}+\vec{\nabla} \cdot(\hat{\vec{u}} \hat{\omega})=0 .
$$

In the two-dimensional Euler equation, we assume a similar hierarchy to the plasma kinetic theory.

\section{EVALUATION OF COLLISION TERM}

Our aim is to evaluate the collisional effect included implicitly in the macroscopic equation corresponding to the microscopic Euler equation [8,9]. The starting equation is the microscopic Euler equation.

$$
\begin{aligned}
& \frac{\partial \hat{\omega}_{+}}{\partial t}+\vec{\nabla} \cdot\left(\hat{\vec{u}} \hat{\omega}_{+}\right)=0, \\
& \frac{\partial \hat{\omega}_{-}}{\partial t}+\vec{\nabla} \cdot\left(\hat{\vec{u}} \hat{\omega}_{-}\right)=0
\end{aligned}
$$

We introduce a double-sign notation and abbreviate the above two equations into the following form.

$$
\frac{\partial \hat{\omega}_{ \pm}}{\partial t}+\vec{\nabla} \cdot\left(\hat{\vec{u}} \hat{\omega}_{ \pm}\right)=0
$$


Microscopic quantity is divided into two parts, a macroscopic part and a fluctuation:

$$
\begin{aligned}
\hat{\omega}_{ \pm} & =\left\langle\hat{\omega}_{ \pm}\right\rangle+\delta \omega_{ \pm}=\omega_{ \pm}+\delta \omega_{ \pm} \\
\hat{\vec{u}} & =\langle\hat{\vec{u}}\rangle+\delta \vec{u}=\vec{u}+\delta \vec{u}
\end{aligned}
$$

Inserting the above expressions (15) and (16) into the microscopic Euler equation (14) and calculating a perturbation expansion, we obtain explicit formulae of $\vec{\Gamma}_{ \pm}$.

$$
\begin{aligned}
& \frac{\partial}{\partial t} \omega_{ \pm}+\vec{\nabla} \cdot\left(\vec{u} \omega_{ \pm}\right)=-\vec{\nabla} \cdot \vec{\Gamma}_{ \pm}(\vec{r}, t) \\
& \vec{\Gamma}_{ \pm}=\left\langle\delta \vec{u} \delta \omega_{ \pm}\right\rangle \\
& =-\int d \vec{r}^{\prime} \vec{F}\left(\vec{r}-\vec{r}^{\prime}\right)\left\langle\delta \omega^{\prime} \delta \omega_{ \pm}\right\rangle \\
& =-\Omega \int d \vec{r}^{\prime} \int \frac{d \vec{k}}{(2 \pi)^{2}} \int \frac{d \vec{k}^{\prime}}{(2 \pi)^{2}} \exp \left(i\left(\vec{k}+\vec{k}^{\prime}\right) \cdot\left(\vec{r}-\vec{r}^{\prime}\right)\right) \pi \delta\left(\vec{k} \cdot\left(\vec{u}-\vec{u}^{\prime}\right)\right) \\
& \times \frac{\hat{\vec{z}} \times i \vec{k}^{\prime}}{|\overrightarrow{\vec{z}} \times i \vec{k}|^{2}} \frac{\left.\vec{k}\right|^{2}}{\mid \vec{k}}\left[\left(\omega_{+}^{\prime}-\omega_{-}^{\prime}\right) \vec{\nabla} \omega_{ \pm} \mp \omega_{ \pm} \vec{\nabla}^{\prime} \omega^{\prime}\right] \\
& \vec{F}(\vec{r})=\hat{\vec{z}} \times \vec{\nabla} G(\vec{r}) .
\end{aligned}
$$

We perform a further coarse-graining to drop the high frequency component $\exp \left(i\left(\vec{k}+\vec{k}^{\prime}\right) \cdot\left(\vec{r}-\vec{r}^{\prime}\right)\right)$ remaining in $\vec{\Gamma}_{ \pm}$. Finally, we obtain the expression of the collision term in separate form:

$$
\begin{gathered}
\frac{\partial}{\partial t} \omega_{ \pm}+\vec{\nabla} \cdot\left(\vec{u} \omega_{ \pm}\right)=-\vec{\nabla} \cdot \vec{\Gamma}_{s \pm}(\vec{r}, t) \\
\vec{\Gamma}_{s \pm}(\vec{r}, t)=-\mathrm{D}_{s} \cdot \vec{\nabla} \omega_{ \pm} \pm \vec{V}_{s} \omega_{ \pm} \\
\mathrm{D}_{s}=K \int d \vec{r}^{\prime} \frac{\left(\vec{u}-\vec{u}^{\prime}\right)\left(\vec{u}-\vec{u}^{\prime}\right)\left(\omega_{+}^{\prime}-\omega_{-}^{\prime}\right)}{\left|\vec{u}-\vec{u}^{\prime}\right|^{3}} \\
\vec{V}_{s}=K \int d \vec{r}^{\prime} \frac{\left(\vec{u}-\vec{u}^{\prime}\right)\left(\vec{u}-\vec{u}^{\prime}\right) \cdot \vec{\nabla}^{\prime} \omega^{\prime}}{\left|\vec{u}-\vec{u}^{\prime}\right|^{3}}
\end{gathered}
$$

or in combined form:

$$
\begin{gathered}
\frac{\partial}{\partial t} \omega+\vec{\nabla} \cdot(\vec{u} \omega)=-\vec{\nabla} \cdot \vec{\Gamma}_{s}(\vec{r}, t), \\
\vec{\Gamma}_{s}(\vec{r}, t)=\vec{\Gamma}_{s+}(\vec{r})+\vec{\Gamma}_{s-}(\vec{r}) \\
=-D_{s} \cdot \vec{\nabla}\left(\omega_{+}+\omega_{-}\right)+\vec{V}_{s}\left(\omega_{+}-\omega_{-}\right) \\
=-K \int d \vec{r}^{\prime} \frac{\left(\vec{u}-\vec{u}^{\prime}\right)\left(\vec{u}-\vec{u}^{\prime}\right)}{\left|\vec{u}-\vec{u}^{\prime}\right|^{3}}\left(\left(\omega_{+}^{\prime}-\omega_{-}^{\prime}\right) \vec{\nabla} \omega-\left(\omega_{+}-\omega_{-}\right) \vec{\nabla}^{\prime} \omega^{\prime}\right)
\end{gathered}
$$

where $K$ is a constant defined by

$$
K=\frac{\Omega}{(2 \pi)^{3}}\left(\frac{\pi}{L}\right)^{2} \frac{1}{k_{\min }}
$$




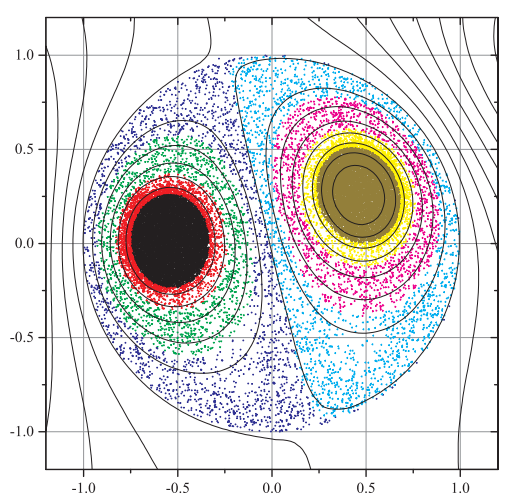

FIGURE 3. Typical equilibrium distribution of the positive and the negative vortices at negative $\beta$ is shown. The distribution is color-coded depending on the density of the vortices.

\section{PHYSICAL PROPERTIES OF COLLISION TERM}

\section{Role of Drift Term}

The drift term drives the positive and the negative vortices in opposite directions. To show this feature, we rewrite the diffusion fluxes in terms of the number density $n_{+}$and $n_{-}$

$$
\begin{gathered}
\omega=\omega_{+}+\omega_{-}=\Omega_{0}\left(n_{+}-n_{-}\right), \\
\vec{\Gamma}_{s+}=\Omega_{0}\left(-\mathrm{D} \cdot \vec{\nabla} n_{+}\right)+\vec{V} n_{+}, \\
\vec{\Gamma}_{s-}=\Omega_{0}\left(-\mathrm{D} \cdot \vec{\nabla} n_{-}\right)-\vec{V} n_{-} .
\end{gathered}
$$

Equations (29) and (30) explicitly indicate the direction of the drift term for the positive vortices is opposite from the one for the negative vortices, while the direction of the diffusion term for the positive vortices is the same as the one for the negative vortices. We find that the drift term provides an essential role for producing the well-known "chargeseparated" equilibrium distribution of the point vortices at negative $\beta$ as is shown in Fig. 3. This is a typical example of the self-organization in the point vortex system at negative temperature. At negative temperature, the like-sign vortices aggregate and make condensates (clumps). The distribution is in thermal equilibrium and is characterized by the sinh-Poisson equation [6]. On the other hand, if temperature is positive, the positive and negative vortices distribute uniformly in the circular wall.

\section{Sign of $d \omega / d \psi$}

Typical equilibrium distribution of the positive and the negative vortices at negative $\beta$ is shown in Fig. 3 . The distribution is color-coded depending on the density of the vortices. We find that the black lines corresponding to the isosurface of the stream function are almost parallel to the boundaries of the regions with different densities. This implies that $\vec{\nabla} \cdot(\vec{u} \omega) \approx 0$ or equivalently $\omega \approx \omega(\psi)$. In a Boltzmann equilibrium state at negative $\beta$, the following relation is satisfied.

$$
\omega_{\mathrm{eq} \pm}=\omega_{0 \pm} \exp \left(\mp \beta \Omega_{0} \psi_{\mathrm{eq}}\right) .
$$

From this equation, we find that the sign of $d \omega_{\mathrm{eq}} / d \psi_{\mathrm{eq}}$ is positive or zero

$$
\begin{aligned}
\frac{d \omega_{\mathrm{eq}}}{d \psi_{\mathrm{eq}}} & =-\beta \Omega_{0}\left(\omega_{\mathrm{eq}+}-\omega_{\mathrm{eq}+}\right) \geq 0, \\
\omega_{\mathrm{eq}} & =\omega_{\mathrm{eq}+}+\omega_{\mathrm{eq}-} .
\end{aligned}
$$


It was anticipated that the relaxation process of the N-body system has two stages by Lynden-Bell [10]. In the point vortex system, similar hierarchy can be considered. The first stage is a collisionless fast process called a violent relaxation whose dynamics are governed by

$$
\frac{\partial \omega}{\partial t}+\vec{\nabla} \cdot(\vec{u} \omega) \approx 0 .
$$

The second stage is a collisional slow process called a slow relaxation whose dynamics are governed by

$$
\frac{\partial \omega}{\partial t} \approx-\vec{\nabla} \cdot \vec{\Gamma}_{s}(\vec{r}, t)
$$

Equation (35) implies that the sign of $d \omega / d \psi$ is expected to be positive or zero during slow relaxation as $\vec{\nabla} \cdot(\vec{u} \omega) \approx 0$. This relation will be used later.

\section{Einstein Relation}

Let us consider a local equilibrium state. In this state, there are many small regions with different temperatures. In each small region, temperature is locally uniform and the following relation is satisfied:

$$
\omega_{\text {leq } \pm}=\omega_{0 \pm} \exp \left(\mp \beta_{\text {leq }} \Omega_{0} \psi_{\text {leq }}\right)
$$

Equation (36) ensures that the integrand of Eq. (26) vanishes. Namely, the detailed balance is established in each small region. Note that $\vec{\Gamma}_{s}(\vec{r}, t)$ does not vanish because correlations with the other small regions with different $\beta$ remain as nonzero. When the system reaches a global equilibrium state with uniform $\beta$, the following relation is satisfied

$$
\omega_{\mathrm{eq} \pm}=\omega_{0 \pm} \exp \left(\mp \beta \Omega_{0} \psi_{\mathrm{eq}}\right),
$$

and the collisional effect vanishes everywhere in the system. In this state, the drift term is rewritten as

$$
\vec{V}=\beta \Omega_{0} \mathrm{D}_{\mathrm{eq}} \cdot \vec{\nabla} \psi_{\mathrm{eq}} \cdot
$$

This equation is a counterpart of the Einstein relation in the point vortex system satisfied in a thermal equilibrium state.

\section{Energy Conservation}

It is shown that the obtained kinetic equation conserves the total mean field energy $E$ :

$$
\begin{gathered}
E \equiv \frac{1}{2} \int d \vec{r} \psi \omega=\frac{1}{2} \int d \vec{r} \int d \vec{r}^{\prime} G\left(\vec{r}-\vec{r}^{\prime}\right) \omega^{\prime} \omega . \\
\frac{d E}{d t}=\frac{1}{2} \int d \vec{r} \int d \vec{r}^{\prime} G\left(\vec{r}-\vec{r}^{\prime}\right)\left(\frac{\partial \omega^{\prime}}{\partial t} \omega+\omega^{\prime} \frac{\partial \omega}{\partial t}\right) \\
=-K \int d \vec{r} \int d \vec{r}^{\prime} \vec{\nabla} \psi \cdot \frac{\left(\vec{u}-\vec{u}^{\prime}\right)\left(\vec{u}-\vec{u}^{\prime}\right)}{\left|\vec{u}-\vec{u}^{\prime}\right|^{3}} \cdot\left[\left(\omega_{+}^{\prime}-\omega_{-}^{\prime}\right) \vec{\nabla} \omega-\left(\omega_{+}-\omega_{-}\right) \vec{\nabla}^{\prime} \omega^{\prime}\right] \\
=-\frac{K}{2} \int d \vec{r} \int d \vec{r}^{\prime}\left(\vec{\nabla} \psi-\vec{\nabla}^{\prime} \psi^{\prime}\right) \cdot \frac{\left(\vec{u}-\vec{u}^{\prime}\right)\left(\vec{u}-\vec{u}^{\prime}\right)}{\left|\vec{u}-\vec{u}^{\prime}\right|^{3}} \cdot\left[\left(\omega_{+}^{\prime}-\omega_{-}^{\prime}\right) \vec{\nabla} \omega-\left(\omega_{+}-\omega_{-}\right) \vec{\nabla}^{\prime} \omega^{\prime}\right] \\
=0 .
\end{gathered}
$$

It is also revealed that the energy conservation is achieved by balancing the energy dissipation process due to the diffusion term and the energy production process due to the drift term.

$$
\begin{aligned}
& \left.\frac{d E}{d t}\right|_{\mathrm{D}}=-K \int d \vec{r} \int d \vec{r}^{\prime} \frac{\left|\vec{\nabla} \psi \cdot\left(\vec{u}-\vec{u}^{\prime}\right)\right|^{2}}{\left|\vec{u}-\vec{u}^{\prime}\right|^{3}}\left(\omega_{+}^{\prime}-\omega_{-}^{\prime}\right) \frac{d \omega}{d \psi} \leq 0, \\
& \left.\frac{d E}{d t}\right|_{\vec{V}}=K \int d \vec{r} \int d \vec{r}^{\prime} \frac{\left|\vec{\nabla} \psi \cdot\left(\vec{u}-\vec{u}^{\prime}\right)\right|^{2}}{\left|\vec{u}-\vec{u}^{\prime}\right|^{3}}\left(\omega_{+}-\omega_{-}\right) \frac{d \omega^{\prime}}{d \psi^{\prime}} \geq 0 .
\end{aligned}
$$




\section{H Theorem}

It can be shown that the $\mathrm{H}$ function is a monotonic decay function with time.

$$
\begin{gathered}
S=-k_{B} H, \\
H=\int d \vec{r} \frac{\omega_{+}}{\Omega} \ln \frac{\omega_{+}}{\Omega}+\frac{\omega_{-}}{-\Omega} \ln \frac{\omega_{-}}{-\Omega}+\text { const. } \\
=\frac{1}{\Omega} \int d \vec{r} \omega_{+} \ln \omega_{+}-\omega_{-} \ln \left|\omega_{-}\right|-2 N \ln \Omega+\text { const. } \\
\frac{d H}{d t=} \frac{1}{\Omega} \int d \vec{r} \frac{\partial \omega_{+}}{\partial t}\left(\ln \omega_{+}+1\right)-\frac{\partial \omega_{-}}{\partial t}\left(\ln \left|\omega_{-}\right|+1\right) \\
=-\frac{K}{\Omega_{0}} \int d \vec{r} \int d \vec{r}^{\prime} \frac{1}{\left|\vec{u}-\vec{u}^{\prime}\right|^{3}}\left[\omega_{+} \omega_{+}^{\prime}\left|\left(\vec{u}-\vec{u}^{\prime}\right) \cdot\left(\vec{\nabla} \ln \omega_{+}+\vec{\nabla} \ln \omega_{+}^{\prime}\right)^{2}\right|\right] \\
-\frac{K}{\Omega_{0}} \int d \vec{r} \int d \vec{r}^{\prime} \frac{1}{\left|\vec{u}-\vec{u}^{\prime}\right|^{3}}\left[\omega_{-} \omega_{-}^{\prime}\left|\left(\vec{u}-\vec{u}^{\prime}\right) \cdot\left(\vec{\nabla} \ln \omega_{-}+\vec{\nabla} \ln \omega_{-}^{\prime}\right)^{2}\right|\right] \\
-\frac{K}{\Omega_{0}} \int d \vec{r} \int d \vec{r}^{\prime} \frac{1}{\left|\vec{u}-\vec{u}^{\prime}\right|^{3}}\left[\omega_{+} \omega_{-}^{\prime}\left|\left(\vec{u}-\vec{u}^{\prime}\right) \cdot\left(\vec{\nabla} \ln \omega_{+}+\vec{\nabla} \ln \omega_{-}^{\prime}\right)^{2}\right|\right] \\
-\frac{K}{\Omega_{0}} \int d \vec{r} \int d \vec{r}^{\prime} \frac{1}{\left|\vec{u}-\vec{u}^{\prime}\right|^{3}}\left[\omega_{-} \omega_{+}^{\prime}\left|\left(\vec{u}-\vec{u}^{\prime}\right) \cdot\left(\vec{\nabla} \ln \omega_{-}+\vec{\nabla} \ln \omega_{+}^{\prime}\right)^{2}\right|\right] \leq 0
\end{gathered}
$$

During the slow relaxation, the diffusion term increases the entropy, while the drift term decreases it.

$$
\begin{aligned}
\left.\frac{d S}{d t}\right|_{\mathrm{D}} & =\frac{k_{B}}{\Omega} \int d \vec{r} \frac{\vec{\nabla} \omega_{+} \cdot \mathrm{D}_{s} \cdot \vec{\nabla} \omega_{+}}{\omega_{+}}-\frac{\vec{\nabla} \omega_{-} \cdot \mathrm{D}_{s} \cdot \vec{\nabla} \omega_{-}}{\omega_{-}} \\
& =k_{B} \frac{K}{\Omega} \int d \vec{r} \int d \vec{r}^{\prime}\left(\frac{\left|\vec{\nabla} \omega_{+} \cdot\left(\vec{u}-\vec{u}^{\prime}\right)\right|^{2}}{\omega_{+}}-\frac{\left|\vec{\nabla} \omega_{-} \cdot\left(\vec{u}-\vec{u}^{\prime}\right)\right|^{2}}{\omega_{-}}\right) \frac{\omega_{+}^{\prime}-\omega_{-}^{\prime}}{\left|\vec{u}-\vec{u}^{\prime}\right|^{3}} \geq 0 \\
\left.\frac{d S}{d t}\right|_{\vec{V}} & =-\frac{k_{B}}{\Omega} \int d \vec{r} \vec{V}_{s} \cdot \vec{\nabla} \omega \\
& =-k_{B} \frac{K}{\Omega} \int d \vec{r} \int d \vec{r}^{\prime} \frac{\left|\vec{\nabla} \psi \cdot\left(\vec{u}-\vec{u}^{\prime}\right)\right|^{2}}{\left|\vec{u}-\vec{u}^{\prime}\right|^{3}} \frac{d \omega}{d \psi} \frac{d \omega^{\prime}}{d \psi^{\prime}} \leq 0
\end{aligned}
$$

These relations clearly indicate that the drift term plays an important role in the clump formation in negative $\beta$ and the key role of the self-organization is provided by the drift term.

\section{NUMERICAL RESULTS}

We will show evidence for the obtained collision term numerically.

Typical time evolution from a non-axisymmetric initial profile is shown in Fig. 4. Time is scaled by dynamical time which is given by a rotation time of a circular clump with initial density inside the rectangular area. The initial, highly asymmetric profile gradually deforms and relaxes toward an axisymmetric profile.

To evaluate the relaxation time, we trace time evolutions of the $H$ function with different coarse-graining scales. The finest coarse-graining scale corresponds to the $R_{0} / 500$ case and the roughest coarse-graining scale the $R_{0} / 2$ case. They are shown in Fig. 5. The figure shows that the $H$ function is a decreasing function with time, if an appropriate coarse-graining scale is given. In this case, the appropriate coarse-graining scale may be between $R_{0} / 5$ and $R_{0} / 25$. As is shown in $R_{0} / 500$ case, the value of the $H$ function is zero throughout the simulation time. This means that at most one vortex is located in a cell for the coarse-graining.

We assume the time dependence of the $\mathrm{H}$ function as follows:

$$
H(t)=H_{0}+H_{1} \exp \left(-\frac{t}{t_{R}}\right)
$$


(a) $0.0 t_{D}$

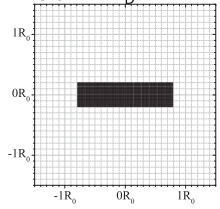

(b) $1.7 t_{D}$

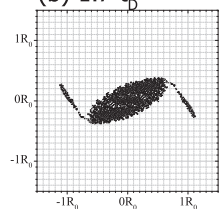

(c) $3.5 t_{p}$

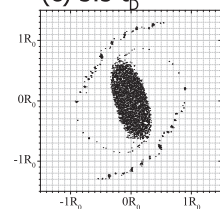

(d) $5.2 t_{D}$

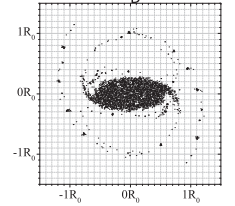

(e) $17 t_{D}$

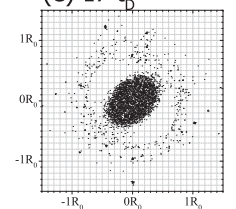

FIGURE 4. Typical time evolution from a non-axisymmetric profile is shown. There are 3751 particles in a rectangle initial profile with $1.6 R_{0} \times 0.4 R_{0}$ where $R_{0}$ is a characteristic system size. Time is scaled by dynamical type which is given by a rotation time of a circular clump with initial density inside the rectangular area.

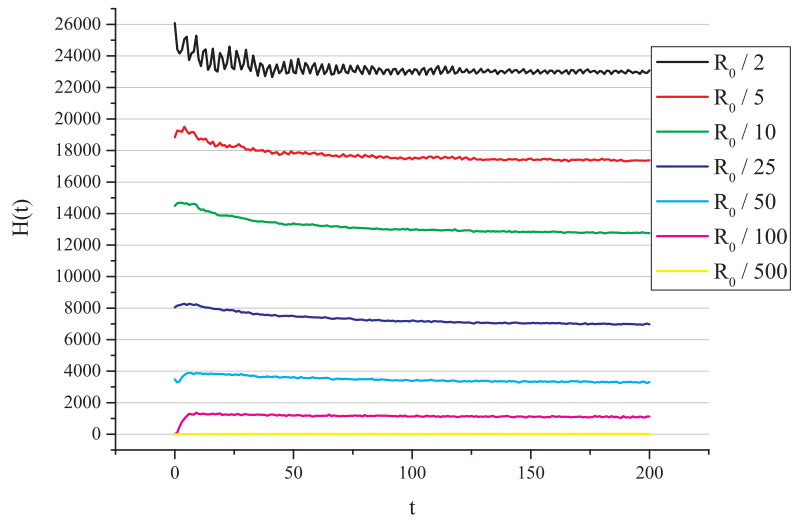

FIGURE 5. Time evolutions of the $H$ function with different coarse-graining scale are shown.

where $t_{R}$ is a characteristic relaxation time. Scalings anticipated by the kinetic equation are given by

$$
\begin{aligned}
t_{R} & \propto \frac{1}{\Omega_{0}}, \\
\frac{t_{R}}{t_{D}} & \propto N .
\end{aligned}
$$

Time evolutions of the $H$ function with different $\Omega_{0}$ and with different $N$ are given in Figs. 6 and 7, respectively.

Relaxation time scale $\tau_{R}$ is determined by the slopes of the $H$ function during the slow relaxation. The relaxation process by the obtained collision term should be characterized not by the violent relaxation but the slow relaxation. The

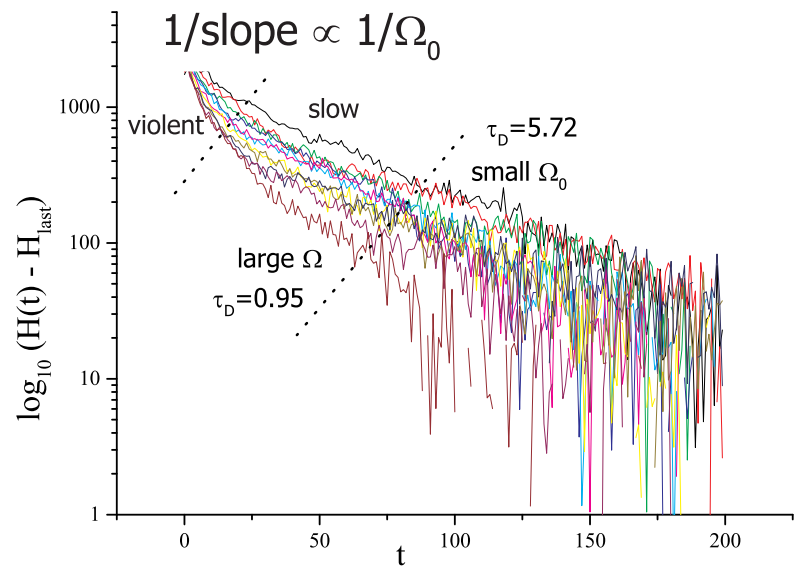

FIGURE 6. Time evolutions of the $H$ function with different circulation $\Omega_{0}$ are shown. 


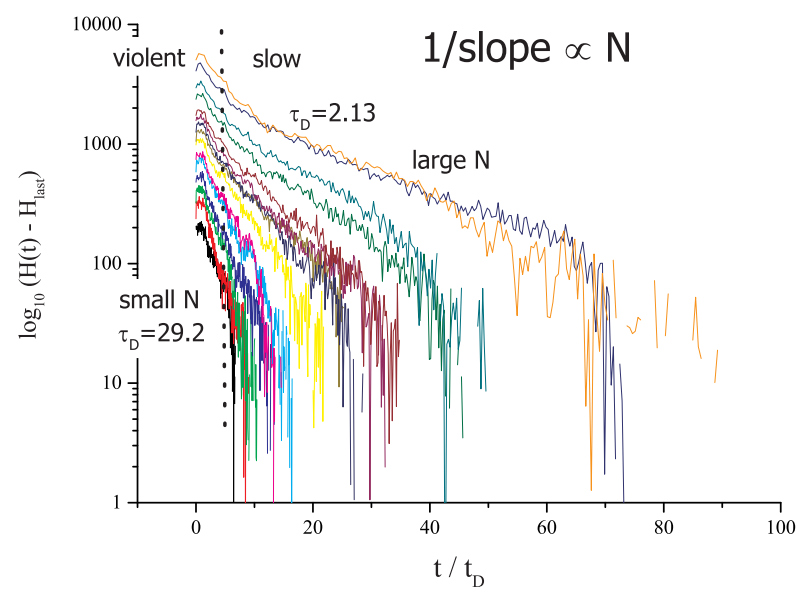

FIGURE 7. Time evolutions of the $H$ function with different number of vortices $N$ are shown.

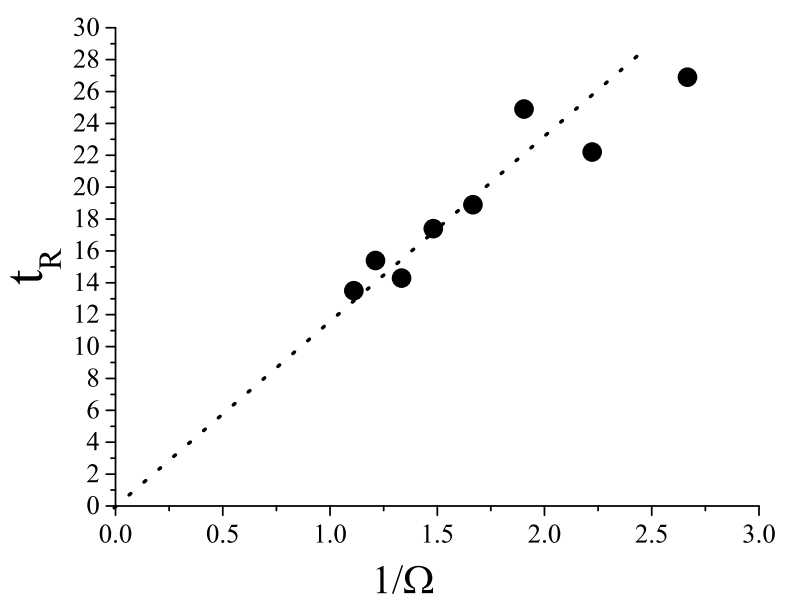

FIGURE 8. Relaxation time is plotted against $1 / \Omega$.

violent relaxation is a rapid process and will last for the several dynamical times from the beginning of the simulation. So, we observe $\tau_{R}$ after $5 \tau_{D}$. Bare relaxation time and the one normalized by the dynamical time $\tau_{D}$ are shown in Fig. 8 and 9, respectively. These figures evidence the theoretical anticipation given in Eqs. (49) and (50).

\section{CONCLUSION}

Kinetic equation with the Fokker-Planck type collision term is derived and the crucial role of the drift term $V$ in 2D self-organization is evidenced. The obtained collision term has several physically good properties. The collision term disappears when the system reaches a Boltzmann equilibrium state. The Einstein relation is derived at the equilibrium state. Conservation of the mean field energy and the $H$ theorem is also derived. The scalings of the relaxation time are confirmed by the numerical simulation.

In the simulations, there are several cases that the relaxation process slows down after the system profile reaches an axially symmetric one. In the beginning of the slow relaxation, the relaxation time scales as $\tau_{R} \propto 1 / \Omega_{0}$. If the product $N \Omega_{0}$ is fixed constant, the relaxation time is proportional to $N$. However, the possibility of the slower relaxation process in a symmetric profile has been pointed out by Chavanis [11, 12]. This may indicate the slower relaxation process whose relaxation time is proportional to $N^{2}$ or higher. 


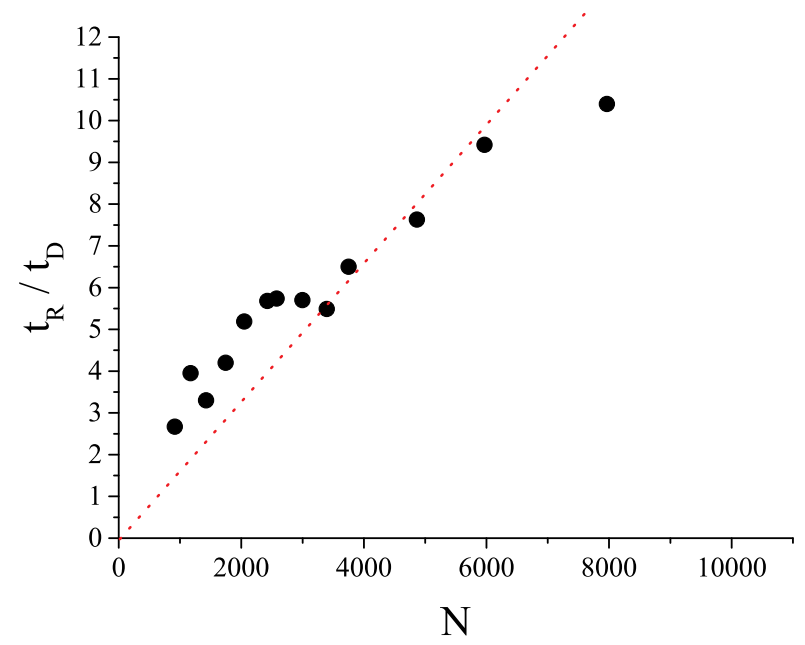

FIGURE 9. Relaxation time normalized by the dynamical time is plotted against $N$.

\section{ACKNOWLEDGMENTS}

The authors are grateful to Prof. P. H. Chavanis for helpful comments. This work was supported by JSPS KAKENHI Grant Number 24540400.

\section{REFERENCES}

1. H. Haken, Synergetics, 3rd Ed., Springer Verlag, Berlin, 1983.

2. A. Sanpei, Y. Kiwamoto, K. Ito, and Y. Soga, Phys. Rev. E 68, 016404 (2003).

3. D. Z. Jin, and D. H. E. Dubin, Phys. Rev. Lett. 80, 4434 (1998).

4. D. A. Schecter, D. H. E. Dubin, K. S. Fine, and C. F. Driscoll, Phys. Fluids 11, 905 (1999).

5. L. Onsager, Nuovo Cimento Suppl. 6, 279 (1949).

6. G. Joyce, and D. Montgomery, J. Plasma Phys. 10, 107-121 (1973).

7. Y. Yatsuyanagi, Y. Kiwamoto, H. Tomita, M. M. Sano, T. Yoshida, and T. Ebisuzaki, Phys. Rev. Lett. 94, 054502 (2005).

8. Y. Yatsuyanagi, T. Hatori, and P. H. Chavanis, J. Phys. Soc. Jpn. 84, 014402 (2015).

9. Y. Yatsuyanagi, and T. Hatori, Arxiv preprint cond-mat.stat-mech (2015), URL http: //arxiv.org/abs /1304 . 7306.

10. D. Lynden-Bell, Mon. Not. R. astr. Soc. 136, 101 (1967).

11. P. H. Chavanis, and M. Lemou, Eur. Phys. J. B 59, 217 (2007).

12. P. H. Chavanis, J. Stat. Mech. 2012, P02019 (2012). 